\title{
Stability of an HIV/AIDS Treatment Model with Different Stages
}

\author{
Hai-Feng Huo and Rui Chen \\ Department of Applied Mathematics, Lanzhou University of Technology, Lanzhou, Gansu 730050, China \\ Correspondence should be addressed to Hai-Feng Huo; hfhuo@lut.cn
}

Received 8 November 2014; Accepted 11 December 2014

Academic Editor: Juan J. Nieto

Copyright (C) 2015 H.-F. Huo and R. Chen. This is an open access article distributed under the Creative Commons Attribution License, which permits unrestricted use, distribution, and reproduction in any medium, provided the original work is properly cited.

\begin{abstract}
An HIV/AIDS treatment model with different stages is proposed in this paper. The stage of the HIV infection is divided into two stages, that is, HIV-positive in the asymptomatic stage of HIV infection and HIV-positive individuals in the pre-AIDS stage. The fact that some individuals with HIV-positive individuals after the treatment can be transformed into the compartment of HIV-positive individuals in the asymptomatic stage of HIV infection, the compartment of HIV-positive individuals in the pre-AIDS stage, or the compartment of individuals with full-blown AIDS is also considered. Mathematical analyses establish the idea that the global dynamics of the HIV/AIDS model are determined by the basic reproduction number $R_{0}$. The disease-free equilibrium is globally asymptotically stable if $R_{0}<1$. The endemic equilibrium is globally asymptotically stable if $R_{0}>1$ for a special case. Numerical simulations are also conducted to support the analytic results.
\end{abstract}

\section{Introduction}

Human immunodeficiency virus (HIV) is one of the major life threatening viruses that are spreading worldwide. AIDS is caused by the human immunodeficiency virus (HIV), which has developed into a global pandemic since the first patient was identified in 1981, making it one of the most destructive epidemics in history $[1,2]$. The HIV prevalence among pregnant women attending antenatal clinics rose from $0.8 \%$ in 1990 to $30.2 \%$ in 2005 and declined slightly to $29.1 \%$ in $2006[3,4]$.

Because of the fact that there is no vaccine, there are many obstacles in the AIDS treatment. Recently, the most prevalent treatment strategy for HIV infected patients is highly active antiretroviral therapies (HAART), which can prolong the lifespans and improve their life quality of infected individuals [5]. Current antiretroviral HIV/AIDS therapy involves the simultaneous administration of two or more antiviral drugs, typically chosen from two major classes: reverse transcriptase inhibitors (RTIs) and protease inhibitors (PIs) [6]. With the help of the antiretroviral therapy, patients can prolong the lifespan during which they are free of HIV-related symptoms and therefore improve their life quality. At least 3.0 millions of people have been saved in the United States, which highlights the significant advances made in HIV disease treatment [7].
That is the reason why the early treatment of AIDS is very meaningful and of great urgency.

It is well known that mathematical modeling is a very important approach to understand the dynamics of any epidemic and to further develop various control and prevention policies $[8,9]$. For the sake of the importance in biological meanings, dynamical properties of HIV/AIDS models and general theory on such dynamical systems have been studied by many authors in recent years [10-13] and references cited therein. They propose mathematical models that described the interactions of the HIV virus, CD4 cells, and CTLs within host, and these mathematical models can provide some insights into the dynamics of HIV viral load in vivo and may play a significant role in the development of a better understanding of HIV/AIDs and drug therapies.

Huo and Chen [14] introduce an HIV/AIDS epidemic model with antiretroviral treatment and establish the idea that the global dynamics of the HIV/AIDS model are determined by the basic reproduction number $R_{0}$. They show that the early treatment of AIDS is necessary and meaningful. But they do not consider the compartments of HIV-positive individuals in the asymptomatic stage of HIV infection and the compartment of HIV-positive individuals in the preAIDS stage not receiving antiretroviral treatment. Yusuf and Benyah [15] present a deterministic model for controlling 
the spread of the disease using change in sexual habits and antiretroviral (ARV) therapy as control measures and formulate a fixed time optimal control problem subject to the model dynamics with the goal of finding the optimal combination of the two control measures that will minimize the cost of the control efforts as well as the incidence of the disease. But they neglect the fact that some individuals with HIV-positive after the treatment can be transformed into the compartment of HIV-positive individuals in the asymptomatic stage of HIV infection, the compartment of HIV-positive individuals in the pre-AIDS stage, or the compartment of individuals with full-blown AIDS.

Motivated by the above, in this paper, we will divide the stage of the HIV infection into two stages, that is, HIVpositive individuals in the asymptomatic stage of HIV infection and HIV-positive individuals in the pre-AIDS stage. We also consider the fact that some individuals with HIV-positive after the treatment can be transformed into the compartment of HIV-positive individuals in the asymptomatic stage of HIV infection, the compartment of HIV-positive individuals in the pre-AIDS stage, or the compartment of individuals with full-blown AIDS.

The organization of this paper is as follows. In the next section, we propose an HIV/AIDS model. In Section 3, the existence and the global stability of equilibria are investigated. Some numerical simulations are given in Section 4. Some discussions are given in last section.

\section{The Model}

2.1. System Description. The total population is divided into six compartments: $S(t), H(t), P(t), A(t), T(t)$, and $R(t)$. $S(t)$ represents the number of susceptible individuals; $H(t)$ represents the number of HIV-positive individuals in the asymptomatic stage of HIV infection; $P(t)$ represents the number of HIV-positive individuals in the pre-AIDS stage not receiving treatment; $A(t)$ represents the number of individuals with full-blown AIDS not receiving treatment; $T(t)$ represents the number of individuals who are receiving ARV treatment; $R(t)$ represents the number of individuals who change their sexual habits and maintain the habits for the rest of their lives. The total number of population at time $t$ is given by

$$
N(t)=S(t)+H(t)+P(t)+A(t)+T(t)+R(t) .
$$

The model structure is shown in Figure 1. The transfer diagram leads to the following system of ordinary differential equations:

$$
\begin{gathered}
\dot{S}=\Lambda-\beta_{1} S H-\beta_{2} S P-d S-\mu_{1} S, \\
\dot{H}=\beta_{1} S H+\beta_{2} S P-k_{1} H-k_{3} H-d H+\left(\alpha_{1}+\alpha_{3}\right) T, \\
\dot{P}=k_{1} H-k_{4} P-k_{2} P-d P+\left(\alpha_{2}+\alpha_{4}\right) T, \\
\dot{A}=k_{2} P+\alpha_{5} T-\left(d+\delta_{1}\right) A,
\end{gathered}
$$

$$
\begin{gathered}
\dot{T}=k_{3} H+k_{4} P-\left(\alpha_{1}+\alpha_{3}\right) T \\
-\left(\alpha_{2}+\alpha_{4}\right) T-\alpha_{5} T-\left(d+\delta_{2}\right) T, \\
\dot{R}=\mu_{1} S-d R .
\end{gathered}
$$

For simplicity, denote $k_{1}+k_{3}+d=a, k_{2}+k_{4}+d=b, \alpha_{1}+$ $\alpha_{2}+\alpha_{3}+\alpha_{4}+\alpha_{5}+d+\delta_{2}=m, \alpha_{1}+\alpha_{3}=h, \alpha_{2}+\alpha_{4}=g$, and $d+\delta_{1}=n$, and then system (2) becomes

$$
\begin{gathered}
\dot{S}=\Lambda-\beta_{1} S H-\beta_{2} S P-d S-\mu_{1} S, \\
\dot{H}=\beta_{1} S H+\beta_{2} S P-a H+h T, \\
\dot{P}=k_{1} H-b P+g T, \\
\dot{A}=k_{2} P+\alpha_{5} T-n A, \\
\dot{T}=k_{3} H+k_{4} P-m T, \\
\dot{R}=\mu_{1} S-d R,
\end{gathered}
$$

where $\Lambda$ is recruitment rate, $d$ is the natural death rate, $\beta_{1}$ is the contact rate between the susceptible and the infected individuals. $\beta_{2}$ is the contact rate between the susceptible and the pre-AIDS stages. $k_{1}$ is the rate at which individuals leave the infection class and become individuals in the preAIDS stage. $k_{2}$ is the rate at which individuals leave the preAIDS class and become individuals with full-blown AIDS. $k_{3}$ is the rate at which an individual in the asymptomatic stage of HIV infection receives treatment. $k_{4}$ is the rate at which an individual in the pre-AIDS stage receives treatment. $\delta_{1}$ and $\delta_{2}$ are the disease-induced death rates for individuals in compartments $A(t)$ and $T(t) . \mu_{1}$ is the rate at which susceptible individuals change their sexual habits per unit time. $\alpha_{1}$ is the rate at which a treated individual leaves compartment $T(t) . \alpha_{2}$ is the rate at which treatment of an individual with HIV fails. $\alpha_{3}$ is the rate at which an individual in the pre-AIDS stage is treated successfully. $\alpha_{4}$ is the rate at which tratment of an individual in the pre-AIDS stage fails. $\alpha_{5}$ is the rate at which treatment of an individual in the preAIDS stage fails. All the parameters can be found in Table 1.

\subsection{Basic Properties}

2.2.1. Invariant Region. It is necessary to prove that all solutions of system (2) with positive initial data will remain positive for all times $t>0$. This will be established by the following lemma.

Lemma 1. The feasible region $\Omega$ defined by

$$
\begin{aligned}
\Omega=\{( & (t), H(t), P(t), A(t), T(t), R(t)) \\
& \in R_{+}^{6} \mid S(t)+H(t) \\
& \left.+P(t)+A(t)+T(t)+R(t) \leq \frac{\Lambda}{d}\right\},
\end{aligned}
$$

with initial condition $S(0) \geq 0, H(0) \geq 0, P(0) \geq 0, A(0) \geq 0$, $T(0) \geq 0$, and $R(0) \geq 0$, is positive invariant for system (2). 


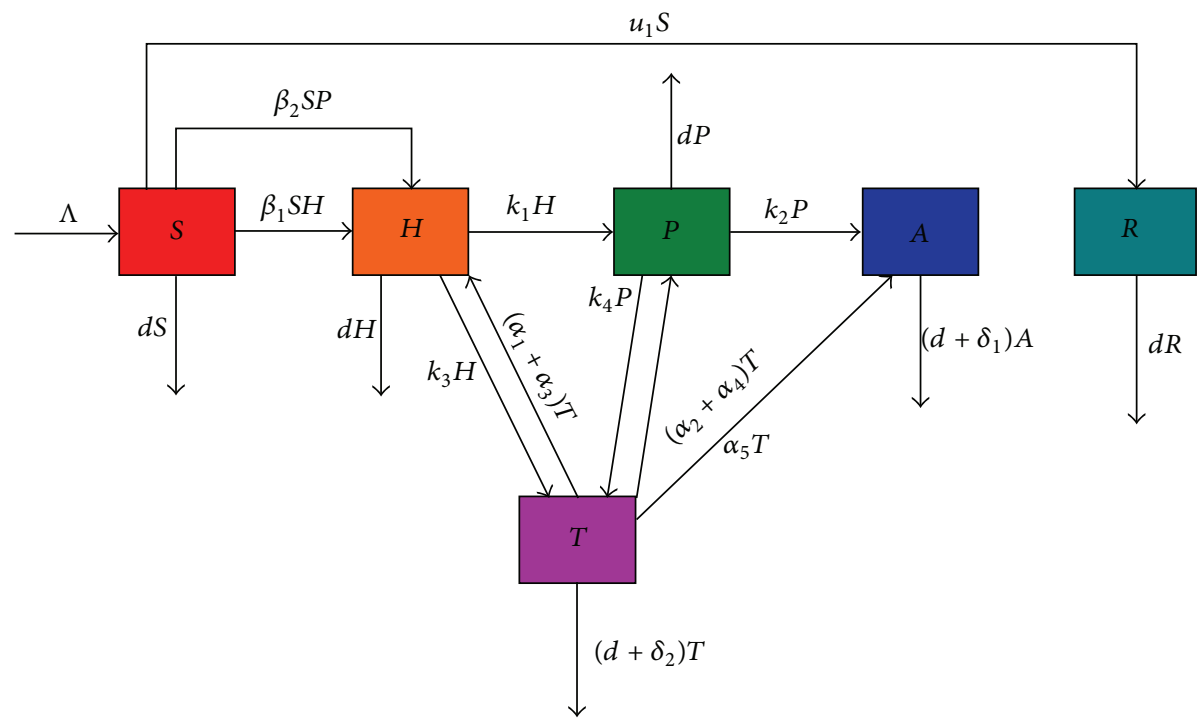

Figure 1: Transfer diagram of the model (2).

TABLE 1: Description and estimation of parameters.

\begin{tabular}{|c|c|c|c|}
\hline Parameter & Description & Estimated value & Date source \\
\hline$\Lambda$ & Recruitment rate of the population & 0.55 year $^{-1}$ & Estimate \\
\hline$\beta_{1}$ & Transmission coefficient for contact with the $\mathrm{H}$ class & 0.3 year $^{-1}$ & Estimate \\
\hline$\beta_{2}$ & Transmission coefficient for contact with the P class & 0.2 year $^{-1}$ & Estimate \\
\hline$d$ & Natural death rate & 0.0196 year $^{-1}$ & {$[15]$} \\
\hline$k_{1}$ & Progression rate from the $\mathrm{H}$ class into the $\mathrm{P}$ class & 0.498 year $^{-1}$ & Estimate \\
\hline$k_{2}$ & Progression rate from the $\mathrm{P}$ class into the A class & 0.08 year $^{-1}$ & Estimate \\
\hline$k_{3}$ & Proportion of the $\mathrm{H}$ class receiving treatment & Variable & Estimate \\
\hline$k_{4}$ & Proportion of the $\mathrm{P}$ class receiving treatment & Variable & Estimate \\
\hline$\alpha_{1}$ & The proportion of the $\mathrm{H}$ class successful treatment & 0.2 year $^{-1}$ & Estimate \\
\hline$\alpha_{2}$ & The proportion of the $\mathrm{P}$ class treatment failure & 0.05 year $^{-1}$ & Estimate \\
\hline$\alpha_{3}$ & The proportion of the $\mathrm{P}$ class successful treatment & 0.15 year $^{-1}$ & Estimate \\
\hline$\alpha_{4}$ & The proportion of the $\mathrm{P}$ class treatment failure & 0.03 year $^{-1}$ & Estimate \\
\hline$\alpha_{5}$ & The proportion of the $\mathrm{P}$ class treatment failure and seriousness & 0.01 year $^{-1}$ & Estimate \\
\hline$\delta_{1}$ & The disease-related death rate of the AIDS & 0.0909 year $^{-1}$ & {$[15]$} \\
\hline$\delta_{2}$ & The disease-related death rate of being treated & 0.0667 year $^{-1}$ & {$[15]$} \\
\hline$\mu_{1}$ & The rate of susceptible individuals who changed their habits & 0.03 year $^{-1}$ & [15] \\
\hline
\end{tabular}

Proof. Adding the equations of system (2), we obtain

$$
\frac{d N}{d t}=\Lambda-d N-\delta_{1} A-\delta_{2} T \leq \Lambda-d N
$$

It follows that

$$
0 \leq N(t) \leq \frac{\Lambda}{d}+N(0) e^{-d t}
$$

where $N(0)$ represents the initial values of the total population. Thus $\lim _{t \rightarrow \infty} \sup N(t) \leq \Lambda / d$. It implies that the region $\Omega=\left\{(S(t), H(t), P(t), A(t), T(t), R(t)) \in R_{+}^{6} \mid S(t)+H(t)+\right.$ $P(t)+A(t)+T(t)+R(t) \leq \Lambda / d\}$ is a positively invariant set for system (2).

\subsubsection{Positivity of Solutions}

Lemma 2. If $S(0) \geq 0, H(0) \geq 0, P(0) \geq 0, A(0) \geq 0, R(0) \geq$ 0 , and $T(0) \geq 0$, the solutions $S(t), H(t), P(t), A(t), T(t)$, and $R(t)$ of system (3) are positive for all $t>0$.

Proof. Under the given initial conditions, we need to prove that the solutions of system (3) are positive [16]; if not, we assume that there is a contradiction: there exists a first time $t_{1}$ such that

$$
\begin{aligned}
& S\left(t_{1}\right)=0, \quad S^{\prime}\left(t_{1}\right)<0, \quad H(t) \geq 0, \\
& P(t) \geq 0, \quad A(t) \geq 0, \quad T(t) \geq 0, \quad R(t) \geq 0, \\
& 0<t<t_{1} \text {, }
\end{aligned}
$$


there exists a $t_{2}$ such that

$$
\begin{array}{rrr}
H\left(t_{2}\right)=0, & H^{\prime}\left(t_{2}\right)<0, & S(t) \geq 0, \\
P(t) \geq 0, \quad A(t) \geq 0, & T(t) \geq 0, & R(t) \geq 0, \\
0<t<t_{2},
\end{array}
$$

there exists a $t_{3}$ such that

$$
\begin{aligned}
& P\left(t_{3}\right)=0, \quad P^{\prime}\left(t_{3}\right)<0, \quad S(t) \geq 0, \\
& H(t) \geq 0, \quad A(t) \geq 0, \quad T(t) \geq 0, \quad R(t) \geq 0, \\
& 0<t<t_{3} \text {, }
\end{aligned}
$$

there exists a $t_{4}$ such that

$$
\begin{array}{rlrl}
A\left(t_{4}\right)=0, & A^{\prime}\left(t_{4}\right)<0, & S(t) \geq 0, \\
H(t) \geq 0, \quad P(t) \geq 0, & T(t) \geq 0, & R(t) \geq 0, \quad(10) \\
& 0<t<t_{4} .
\end{array}
$$

there exists a $t_{5}$ such that

$$
\begin{array}{rrr}
T\left(t_{5}\right)=0, & T^{\prime}\left(t_{5}\right)<0, & S(t) \geq 0, \\
H(t) \geq 0, \quad P(t) \geq 0, & A(t) \geq 0, & R(t) \geq 0, \\
& 0<t<t_{5},
\end{array}
$$

and there exists a $t_{6}$ such that

$$
\begin{array}{rrr}
R\left(t_{6}\right)=0, \quad R^{\prime}\left(t_{6}\right)<0, & S(t) \geq 0, \\
H(t) \geq 0, \quad P(t) \geq 0, & A(t) \geq 0, \quad T(t) \geq 0, \\
& & 0<t<t_{6} .
\end{array}
$$

In the first case, we have

$$
S^{\prime}\left(t_{1}\right)=\Lambda>0,
$$

which is a contradiction, so that we can derive that $S(t) \geq 0$, $t \geq 0$. In the second case, we have

$$
H^{\prime}\left(t_{2}\right)=\left(\alpha_{1}+\alpha_{3}\right) T\left(t_{2}\right)+\beta_{2} S\left(t_{2}\right) P\left(t_{2}\right) \geq 0,
$$

which is a contradiction, so that we can derive that $H(t) \geq 0$, $t \geq 0$. Similarly, it can be shown that $P(t) \geq 0, A(t) \geq 0$, $T(t) \geq 0$, and $R(t) \geq 0$ for all $t \geq 0$.

Thus, the solutions $S(t), H(t), P(t), A(t), T(t)$, and $R(t)$ of system (3) remain positive for all $t>0$.

\section{Analysis of the Model}

There exist one disease-free equilibrium $E_{0}$ and one endemic equilibrium $E^{*}$ for system (3).

3.1. Disease-Free Equilibrium and the Reproduction Number. The model has a disease-free equilibrium given by

$$
E_{0}=\left(\frac{\Lambda}{\mu_{1}+d}, 0,0,0,0, \frac{\Lambda \mu_{1}}{d\left(\mu_{1}+d\right)}\right) .
$$

In the following, the basic reproduction number of system (3) will be obtained by the next generation matrix method formulated in $[17,18]$.

Letting $x=(H, P, A, T, S, R)^{T}$, then system (3) can be written as

$$
\frac{d x}{d t}=\mathscr{F}(x)-\mathscr{V}(x),
$$

where

$$
\mathscr{F}(x)=\left(\begin{array}{c}
\beta_{1} S H+\beta_{2} S P \\
0 \\
0 \\
0 \\
0 \\
0
\end{array}\right) \text {, }
$$

$$
\mathscr{V}(x)=\left(\begin{array}{c}
a H-h T \\
b P-g T-k_{1} H \\
n A-k_{2} P-\alpha_{5} T \\
m T-k_{3} H-k_{4} P \\
\beta_{1} S H+\beta_{2} S P+d S+\mu_{1} S-\Lambda \\
d R-\mu_{1} S
\end{array}\right) .
$$

The Jacobian matrices of $\mathscr{F}(x)$ and $\mathscr{V}(x)$ at the free equilibrium $E_{0}$ are, respectively,

$$
\begin{aligned}
& D \mathscr{F}\left(E_{0}\right)=\left(\begin{array}{ccc}
F_{4 \times 4} & 0 & 0 \\
0 & 0 & 0 \\
0 & 0 & 0
\end{array}\right), \\
& D \mathscr{V}\left(E_{0}\right)=\left(\begin{array}{ccccccc}
V_{4 \times 4} & & & & 0_{4 \times 1} & 0_{4 \times 1} \\
\frac{\beta_{1} \Lambda}{d+\mu_{1}} & \frac{\beta_{2} \Lambda}{d+\mu_{1}} & 0 & 0 & d+\mu_{1} & -\mu_{1} \\
0 & 0 & 0 & 0 & & d
\end{array}\right) \text {, }
\end{aligned}
$$

where

$$
\begin{gathered}
F=\left(\begin{array}{cccc}
\frac{\beta_{1} \Lambda}{d+\mu_{1}} & \frac{\beta_{2} \Lambda}{d+\mu_{1}} & 0 & 0 \\
0 & 0 & 0 & 0 \\
0 & 0 & 0 & 0 \\
0 & 0 & 0 & 0
\end{array}\right), \\
V=\left(\begin{array}{cccc}
a & 0 & 0 & -h \\
-k_{1} & b & 0 & -g \\
0 & -k_{2} & n & -\alpha_{5} \\
-k_{3} & -k_{4} & 0 & m
\end{array}\right) .
\end{gathered}
$$

The model reproduction number, denoted by $R_{0}$, is thus given by

$$
R_{0}=\rho\left(F V^{-1}\right)=\frac{\beta_{1} \Lambda\left(b m-k_{4} g\right)+\beta_{2} \Lambda\left(k_{1} m+k_{3} g\right)}{\left(d+\mu_{1}\right)\left(a b m-k_{1} k_{4} h-k_{3} h b-k_{4} g a\right)} .
$$

\subsection{Global Stability of $E_{0}$}

Theorem 3. The disease-free equilibrium $E_{0}$ is globally asymptotically stable for $R_{0}<1$ and unstable otherwise. 
Proof. According to $[19,20]$, we introduce the Lyapunov candidate function

$$
V(t)=H+\Theta P+c T, \quad \Theta>0, c>0 .
$$

Differentiating $V(t)$ with respect to time yields

$$
\dot{V}=\dot{H}+\Theta \dot{P}+c \dot{T} .
$$

Substituting the model equations into (22), we get

$$
\begin{aligned}
\dot{V}= & \beta_{1} S H+\beta_{2} S P-a H+h T \\
& +\Theta\left(k_{1} H-b P+g T\right)+c\left(k_{3} H+k_{4} P-m T\right) .
\end{aligned}
$$

With $S_{0}=\Lambda /\left(d+\mu_{1}\right)$ and $\bar{R}_{0}=\Lambda \mu_{1} / d\left(d+\mu_{1}\right)$, we have

$$
\begin{aligned}
\dot{V} \leq & \frac{\beta_{1} \Lambda H}{d+\mu_{1}}+\frac{\beta_{2} \Lambda P}{d+\mu_{1}}-a H+h T \\
& +\Theta\left(k_{1} H-b P+g T\right)+c\left(k_{3} H+k_{4} P-m T\right) \\
= & \left(\frac{\beta_{1} \Lambda}{d+\mu_{1}}-a+k_{1} \Theta+c k_{3}\right) H \\
& +\left(\frac{\beta_{2} \Lambda}{d+\mu_{1}}-b \Theta+c k_{4}\right) P+(h+\Theta g-c m) T .
\end{aligned}
$$

In order to get the value of $c$ and $\Theta$, we can use

$$
\begin{gathered}
\frac{\beta_{2} \Lambda}{d+\mu_{1}}-b \Theta+c k_{4}=0, \\
h+\Theta g-c m=0 .
\end{gathered}
$$

From (25), we can calculate

$$
c=\frac{\beta_{2} \Lambda g+h b\left(d+\mu_{1}\right)}{\left(b m-k_{4} g\right)\left(d+\mu_{1}\right)}, \quad \Theta=\frac{\beta_{2} \Lambda m+k_{4} h\left(d+\mu_{1}\right)}{\left(b m-k_{4} g\right)\left(d+\mu_{1}\right)} .
$$

Taking $c$ and $\Theta$ into (24), we can get

$$
\begin{gathered}
\dot{V} \leq\left[\frac{\beta_{1} \Lambda}{d+\mu_{1}}-a+\frac{k_{1} \beta_{2} \Lambda m+k_{1} k_{4} h\left(d+\mu_{1}\right)}{\left(b m-k_{4} g\right)\left(d+\mu_{1}\right)}\right. \\
\left.+\frac{\beta_{2} \Lambda g k_{3}+h b k_{3}\left(d+\mu_{1}\right)}{\left(b m-k_{4} g\right)\left(d+\mu_{1}\right)}\right] H \\
=\left[\left(\frac{\beta_{1} \Lambda}{d+\mu_{1}}\left(b m-k_{4} g\right)+\frac{\beta_{2} \Lambda}{d+\mu_{1}}\left(m k_{1}+g k_{3}\right)\right.\right. \\
\left.\quad-a b m+a k_{4} g+k_{1} k_{4} h+k_{3} h b\right) \\
\left.\cdot\left(b m-k_{4} g\right)^{-1}\right] H .
\end{gathered}
$$

Since

$$
R_{0}=\rho\left(F V^{-1}\right)=\frac{\beta_{1} \Lambda\left(b m-k_{4} g\right)+\beta_{2} \Lambda\left(k_{1} m+k_{3} g\right)}{\left(d+\mu_{1}\right)\left(a b m-k_{1} k_{4} h-k_{3} h b-k_{4} g a\right)},
$$

we can obtain

$$
\begin{gathered}
R_{0}-1=\left(\frac{\beta_{1} \Lambda}{d+\mu_{1}}\left(b m-k_{4} g\right)+\frac{\beta_{2} \Lambda}{d+\mu_{1}}\left(m k_{1}+g k_{3}\right)\right. \\
\left.-a b m+a k_{4} g+k_{1} k_{4} h+k_{3} h b\right)
\end{gathered}
$$

$$
\cdot\left(a b m-k_{1} k_{4} h-k_{3} h b-k_{4} g a\right)^{-1}
$$

when $R_{0} \leq 1$,

$$
\dot{V} \leq\left[\frac{\left(a b m-k_{1} k_{4} h-k_{3} h b-k_{4} g a\right)\left(R_{0}-1\right)}{b m-k_{4} g}\right] H \leq 0 .
$$

While $\dot{V}=0$, only $H=T=P=0$. Substituting $H=$ $T=P=0$ into system (3), we can have $S \rightarrow \Lambda /(d+$ $\left.\mu_{1}\right), A \rightarrow 0$, and $R \rightarrow \Lambda \mu_{1} / d\left(d+\mu_{1}\right)$ as $t \rightarrow \infty$. According to LaSalle's invariance principle [21], the diseasefree equilibrium is globally asymptotically stable.

\subsection{Endemic Equilibrium}

3.3.1. Existence of the Endemic Equilibrium. If $R_{0}>1$, system (3) has a unique endemic equilibrium $E^{*}\left(S^{*}, H^{*}, P^{*}, A^{*}, T^{*}\right.$, $R^{*}$ ), where

$$
\begin{gathered}
S^{*}=\frac{\Lambda}{R_{0}\left(\mu_{1}+d\right)}, \\
H^{*}=\frac{\left(b m-g k_{4}\right) P^{*}}{g k_{3}+m k_{1}}, \\
P^{*}=\frac{\left(R_{0}-1\right)\left(\mu_{1}+d\right)\left(g k_{3}+m k_{1}\right)}{\beta_{1}\left(b m-g k_{4}\right)+\beta_{2}\left(g k_{3}+m k_{1}\right)}, \\
A^{*}=\frac{k_{2} P^{*}+\alpha_{5} T^{*}}{n}, \\
T^{*}=\frac{\left(b k_{3}+k_{1} k_{4}\right) P^{*}}{g k_{3}+m k_{1}}, \\
R^{*}=\frac{\mu_{1} \Lambda}{d R_{0}\left(\mu_{1}+d\right)} .
\end{gathered}
$$

\subsubsection{Global Stability of the Endemic Equilibrium}

Theorem 4. If $R_{0}>1, k_{3}=0$, the endemic equilibrium $E^{*}$ is globally asymptotically stable. 
Proof. To study the globally asymptotic stability of the endemic equilibrium, motivated by $[22,23]$, we use a Lyapunov function $V$ as follows:

$$
\begin{aligned}
V= & \left(S-S^{*} \ln S\right)+B\left(H-H^{*} \ln H\right)+C\left(P-P^{*} \ln P\right) \\
& +D\left(T-T^{*} \ln T\right) .
\end{aligned}
$$

Applying the replacements

$$
\frac{S}{S^{*}}=x, \quad \frac{H}{H^{*}}=y, \quad \frac{P}{P^{*}}=z, \quad \frac{T}{T^{*}}=\mu,
$$

the derivative of $V$ is given by

$$
\begin{aligned}
\dot{V}= & \frac{d S}{d t}-\frac{S^{*} d S}{S d t}+B\left(\frac{d H}{d t}-\frac{H^{*} d H}{H d t}\right) \\
& +C\left(\frac{d P}{d t}-\frac{P^{*} d P}{P d t}\right)+D\left(\frac{d T}{d t}-\frac{T^{*} d T}{T d t}\right) \\
= & \frac{d S}{d t}\left(1-\frac{S^{*}}{S}\right)+B\left(1-\frac{H^{*}}{H}\right) \frac{d H}{d t}+C\left(1-\frac{P^{*}}{P}\right) \frac{d P}{d t} \\
& +D\left(1-\frac{T^{*}}{T}\right) \frac{d T}{d t} \\
= & \left(1-\frac{1}{x}\right)\left(\Lambda-\beta_{1} S H-\beta_{2} S P-d S-\mu_{1} S\right) \\
& +B\left(1-\frac{1}{y}\right)\left(\beta_{1} S H+\beta_{2} S P-a H+h T\right) \\
& +C\left(1-\frac{1}{z}\right)\left(k_{1} H-b P+g T\right) \\
& +D\left(1-\frac{1}{\mu}\right)\left(k_{4} P-m T\right) .
\end{aligned}
$$

Since $E(t)$ satisfies the equation of the model with system (3),

$$
\begin{gathered}
\Lambda-\beta_{1} S^{*} H^{*}-\beta_{2} S^{*} P^{*}-d S^{*}-\mu_{1} S^{*}=0, \\
\beta_{1} S^{*} H^{*}+\beta_{2} S^{*} P^{*}-a H^{*}+h T^{*}=0, \\
k_{1} H^{*}-b P^{*}+g T^{*}=0, \\
k_{2} P^{*}+\alpha_{5} T^{*}-n A^{*}=0, \\
k_{3} H^{*}+k_{4} P^{*}-m T^{*}=0, \\
\mu_{1} S^{*}-d R^{*}=0, \\
\dot{V}=\left(1-\frac{1}{x}\right)\left(\beta_{1} S^{*} H^{*}+\beta_{2} S^{*} P^{*}+d S^{*}+\mu_{1} S^{*}\right. \\
\left.-\beta_{1} S^{*} H^{*} x y-\beta_{2} S^{*} P^{*} x z-d S^{*} x-\mu_{1} S^{*} x\right) \\
+B\left(1-\frac{1}{y}\right)\left(\beta_{1} S^{*} H^{*} x y+\beta_{2} S^{*} P^{*} x z\right. \\
\left.-a H^{*} y+h T^{*} \mu\right)
\end{gathered}
$$

$$
\begin{aligned}
& +C\left(1-\frac{1}{z}\right)\left(k_{1} H^{*} y-b P^{*} z+g T^{*} \mu\right) \\
& +D\left(1-\frac{1}{\mu}\right)\left(k_{4} P^{*} z-m T^{*} \mu\right) \\
& =-\frac{(1-x)^{2}}{x}\left(d+\mu_{1}\right) S^{*} \\
& +\left[\beta_{1} S^{*} H^{*}(1-x y)+\beta_{2} S^{*} P^{*}(1-x z)\right]
\end{aligned}
$$$$
\cdot\left(1-\frac{1}{x}\right)
$$$$
+B\left(1-\frac{1}{y}\right)\left(\beta_{1} S^{*} H^{*} x y+\beta_{2} S^{*} P^{*} x z\right.
$$$$
-\frac{\beta_{1} S^{*} H^{*}+\beta_{2} S^{*} P^{*}+h T^{*}}{H^{*}} H^{*} y
$$$$
\left.+h T^{*} \mu\right)
$$$$
+C\left(1-\frac{1}{z}\right)\left(k_{1} H^{*} y-\frac{k_{1} H^{*}+g T^{*}}{P^{*}} P^{*} z+g T^{*} \mu\right)
$$$$
+D\left(1-\frac{1}{\mu}\right)\left(k_{4} P^{*} z-\frac{k_{4} P^{*}}{T^{*}} T^{*} \mu\right)
$$$$
=-\frac{(1-x)^{2}}{x}\left(d+\mu_{1}\right) S^{*}+\beta_{1} S^{*} H^{*}\left(1-x y-\frac{1}{x}+y\right)
$$$$
+\beta_{2} S^{*} P^{*}\left(1-x z-\frac{1}{x}+z\right)
$$$$
+B\left(1-\frac{1}{y}\right)
$$$$
\cdot\left[\beta_{1} S^{*} H^{*}(x y-y)+\beta_{2} S^{*} P^{*}(x z-y)\right.
$$

$$
\begin{aligned}
& \left.+h T^{*}(\mu-y)\right] \\
+ & C\left(1-\frac{1}{z}\right)\left[k_{1} H^{*}(y-z)+g T^{*}(\mu-z)\right] \\
+ & D k_{4} P^{*}\left(1-\frac{1}{\mu}\right)(z-\mu) \\
= & \frac{(1-x)^{2}}{x}\left(d+\mu_{1}\right) S^{*} \\
+ & \beta_{1} S^{*} H^{*}\left(1-x y-\frac{1}{x}+y\right) \\
+ & \beta_{2} S^{*} P^{*}\left(1-x z-\frac{1}{x}+z\right) \\
+ & B \beta_{1} S^{*} H^{*}(x y-x-y+1) \\
+ & B \beta_{2} S^{*} P^{*}\left(x z-\frac{x z}{y}-y+1\right) \\
+ & B h T^{*}\left(\mu-y-\frac{\mu}{y}+1\right)
\end{aligned}
$$




$$
\begin{aligned}
+ & C k_{1} H^{*}\left(y-z-\frac{y}{z}+1\right)+C g T^{*}\left(\mu-z-\frac{\mu}{z}+1\right) \\
+ & D k_{4} P^{*}\left(z-\mu-\frac{z}{\mu}+1\right) \\
= & \frac{(1-x)^{2}}{x}\left(d+\mu_{1}\right) S^{*}+(B-1) \beta_{1} S^{*} H^{*} x y \\
+ & (B-1) \beta_{2} S^{*} P^{*} x z \\
+ & \left(\beta_{1} S^{*} H^{*}-B \beta_{1} S^{*} H^{*}-B \beta_{2} S^{*} P^{*}\right. \\
& \left.-B h T^{*}+C k_{1} H^{*}\right) y \\
+ & \left(\beta_{2} S^{*} P^{*}-C k_{1} H^{*}\right) z \\
+ & \left(-C g T^{*}+D k_{4} P^{*}\right) z+\left(B h T^{*}+C g T^{*}-D k_{4} P^{*}\right) \mu \\
+ & (1+B) \beta_{1} S^{*} H^{*}+(1+B) \beta_{2} S^{*} P^{*} \\
+ & B h T^{*}+C k_{1} H^{*}+C g T^{*}+D k_{4} P^{*}-\frac{\beta_{1} S^{*} H^{*}}{x} \\
+ & \frac{\mu}{z} C g T^{*}-\frac{z}{\mu} D k_{4} P^{*} . \\
- & B \beta_{1} S^{*} H^{*} x-\frac{\beta_{2} S^{*} P^{*}}{x} \\
& \frac{x z}{y} B \beta_{2} S^{*} P^{*}-B h T^{*} \frac{\mu}{z} C k_{1} H^{*} \\
& \\
& \\
&
\end{aligned}
$$

We choose the coefficients of $x y, x z, y, z$, and $\mu$ which are equal to zero; that is,

$$
\begin{gathered}
B-1=0, \\
B h T^{*}+C g T^{*}-D k_{4} P^{*}=0, \\
\beta_{2} S^{*} P^{*}-C k_{1} H^{*}-C g T^{*}+D k_{4} P^{*}=0, \\
\beta_{1} S^{*} H^{*}-B \beta_{1} S^{*} H^{*}-B \beta_{2} S^{*} P^{*}-B h T^{*}+C k_{1} H^{*}=0 .
\end{gathered}
$$

From (36), we have

$$
\begin{gathered}
B=1, \quad C=\frac{\beta_{2} S^{*} P^{*}+h T^{*}}{k_{1} H^{*}}, \\
D=\frac{k_{1} H^{*} h T^{*}+\left(\beta_{2} S^{*} P^{*}+h T^{*}\right) g T^{*}}{k_{1} k_{4} H^{*} P^{*}} .
\end{gathered}
$$

From (37), the derivative of $V$ is given by

$$
\begin{aligned}
\dot{V}= & -\frac{(1-x)^{2}}{x}\left(d+\mu_{1}\right) S^{*}+2 \beta_{1} S^{*} H^{*}+2 \beta_{2} S^{*} P^{*} \\
& +h T^{*}+C k_{1} H^{*}+C g T^{*}+D k_{4} P^{*}-\frac{\beta_{1} S^{*} H^{*}}{x} \\
& -\beta_{1} S^{*} H^{*} x-\frac{\beta_{2} S^{*} P^{*}}{x}-\frac{x z}{y} \beta_{2} S^{*} P^{*}
\end{aligned}
$$

$$
\begin{aligned}
& -h T^{*} \frac{\mu}{y}-\frac{y}{z} C k_{1} H^{*}-\frac{\mu}{z} C g T^{*}-\frac{z}{\mu} D k_{4} P^{*} \\
= & \left(2-x-\frac{1}{x}\right) \beta_{1} S^{*} H^{*}+\left(3-\frac{1}{x}-\frac{x z}{y}-\frac{y}{z}\right) \beta_{2} S^{*} P^{*} \\
& +\left(3-\frac{y}{z}-\frac{\mu}{y}-\frac{z}{\mu}\right) h T^{*}+\left(2-\frac{\mu}{z}-\frac{z}{\mu}\right) C g T^{*}
\end{aligned}
$$$$
\leq 0 \text {. }
$$

According to [24], since the arithmetical mean is greater than or equal to the geometrical mean, then $2-x-1 / x \leq 0$ for $x>0$ and $2-x-1 / x=0$ if and only if $x=1 ; 3-1 / x-x z / y-y / z=0$ for $x>0, y>0$, and $z>0$ if and only if $x=y=z=1$, and $3-y / z-\mu / y-z / \mu=0$ if and only if $\mu=y=z$. Futhermore, $\dot{V}=0$ if and only if $x=y=z=\mu=1$. The maximum invariant set of system (3) on the set $\{(x, y, z, \mu)$ : $\dot{V}=0\}$ is the singleton $(1,1,1,1)$. Thus, for system (3), the endemic equilibrium is globally asymptotically stable if $R_{0}>$ 1 by LaSalle invariance principle.

Remark 5. Biologically speaking, $k_{3}=0$ implies that the $\mathrm{HIV}$-positive individuals in the asymptomatic stage of HIV infection do not receive treatment. Theorem 4 tells us that when HIV-positive individuals in the asymptomatic stage of HIV infection do not receive treatment, the endemic equilibrium $E^{*}$ is globally asymptotically stable. This means that AIDS is persistent. When $k_{3} \neq 0$, the same result is validated by numerical simulations (see Figure 4 ).

\section{Numerical Simulation}

In this section, we present some numerical simulations of system (3) to illustrate our results. To illustrate the analytic results obtained above, we give some simulations using the parameter values in Table 1 .

First, when $k_{3} \neq 0$, we choose $k_{3}=0.6, k_{4}=0.4$, numerical simulation gives $R_{0}=0.95047<1$, and the disease-free equilibrium $E_{0}$ is globally asymptotically stable (see Figure 2). Figure 2 indicates the effect of treatment on stability of the equilibria of the HIV/AIDS infection model. At first, The number of patients who do not receive treatment is increasing constantly. After treatment, The number of patients is decreasing and tends to be zero at the end, which indicates that the spread of infection can be slowed down.

Second, we choose $k_{3}=0.51086, k_{4}=0.4$, numerical simulation gives $R_{0}=1$, and the disease-free equilibrium $E_{0}$ is globally asymptotically stable (Figure 3 ). If we give a proper treatment, the number of individuals with AIDS/HIV will be controlled to some extent. Hence, the disease will be eradicated in the future.

Third, we choose $k_{3}=0.05, k_{4}=0.4$, numerical simulation gives $R_{0}=1.412$, and the endemic equilibrium is globally asymptotically stable (Figure 4 ). Figure 4 indicates that when the basic reproduction is greater than one, as time goes on, the number of people finally tends to be constant, which means that the endemic equilibrium is globally asymptotically stable. 


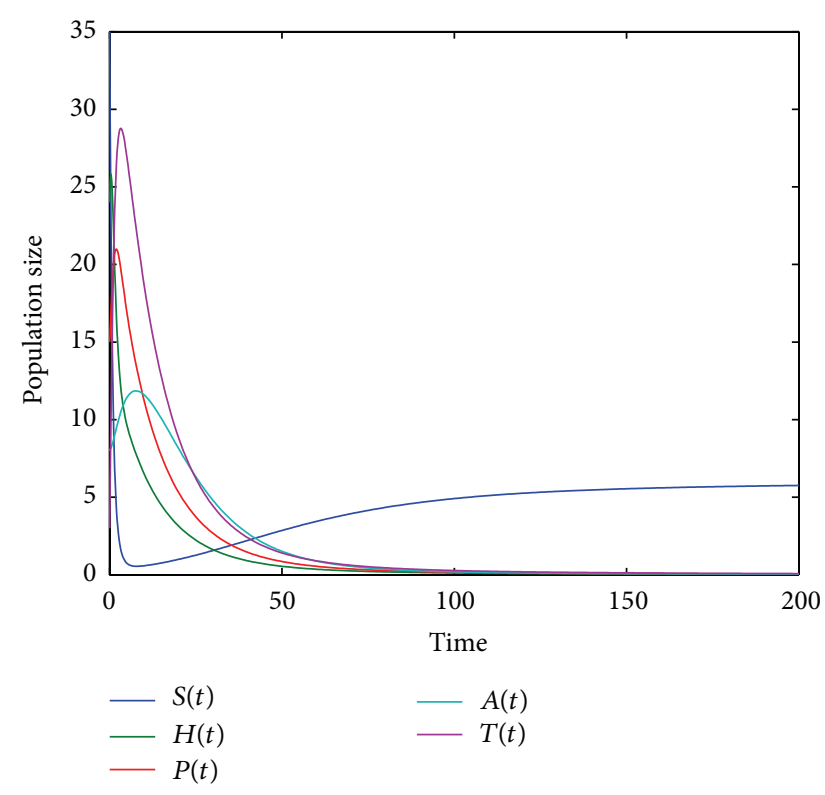

FIGURE 2: When $k_{3}=0.6, k_{4}=0.4$, and $R_{0}=0.95047<1$, the disease-free equilibrium is globally asymptotically stable.

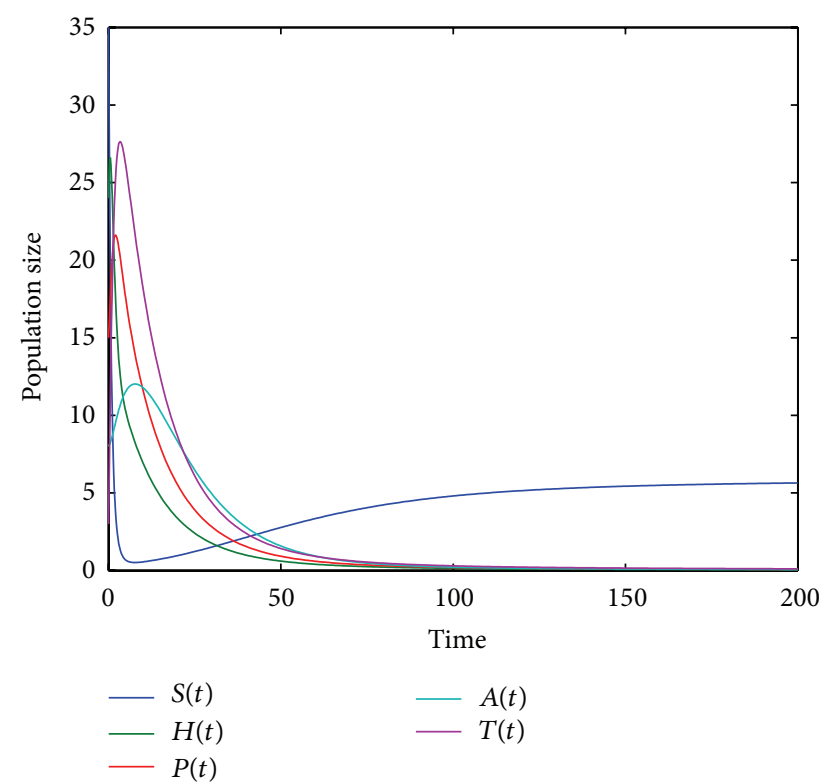

FIgURE 3: When $k_{3}=0.51086, k_{4}=0.4$, and $R_{0}=1$, the disease-free equilibrium is globally asymptotically stable.

Finally, we show the relation between $k_{3}, k_{4}$, and $R_{0}$. When the value of $k_{3}$ and $k_{4}$ is increasing at the time, the basic reproduction is reducing sharply. What is more, Figure 5 confirms that, with the increase in the rate of treatment, the number of individuals with HIV-positive and full-blown AIDS but not receiving treatment and who are receiving ARV treatment tends to zero, which indicates that treatment is important and can prolong their lives.

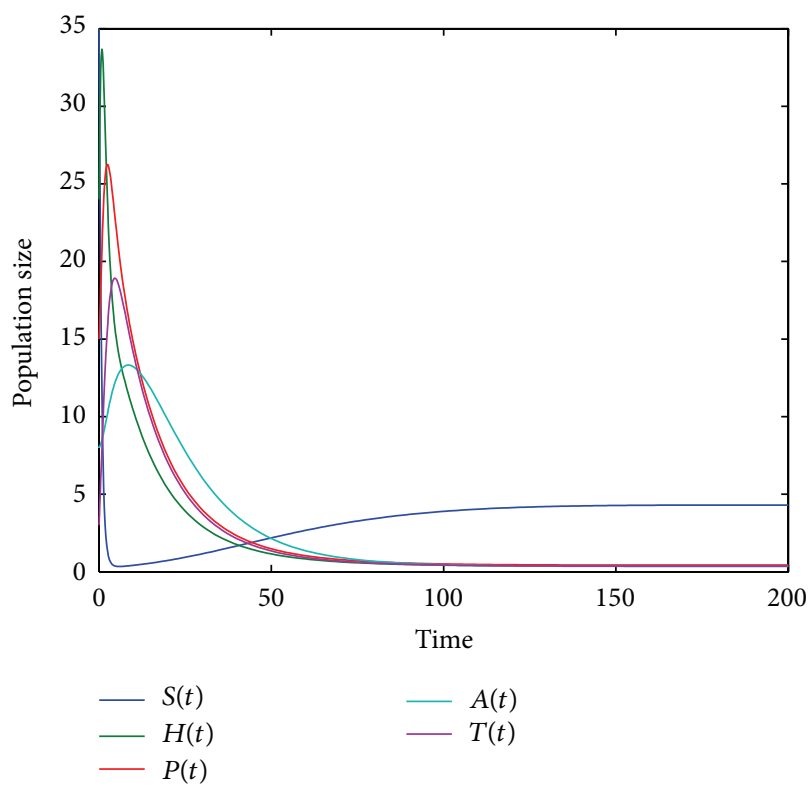

Figure 4: When $k_{3}=0.05, k_{4}=0.4$, and $R_{0}=1.412>1$, the endemic equilibrium is globally asymptotically stable.

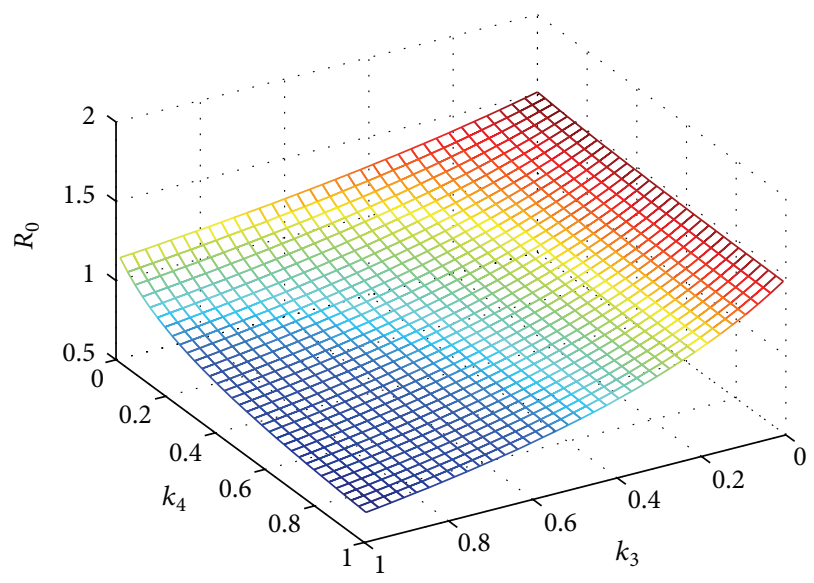

Figure 5: The relationship among $R_{0}, k_{3}$, and $k_{4}$.

\section{Discussion}

A mathematical model is proposed and analyzed to study the spread of HIV/AIDS with treatment. In this paper we have analyzed a stage structured model for HIV and the effect of treatment has also been studied. In the presented model, we get two equilibria: the disease-free equilibrium and the endemic equilibrium. We further consider global asymptotic stability of the disease-free equilibrium by using the wellknown Lyapunov-LaSalle invariance principal. It is found that the disease-free equilibrium is globally asymptotically stable when the basic reproduction number is less than one. When the basic reproduction number is greater than one and $k_{3}=0$, the endemic equilibrium is globally asymptotically stable. Our results show that early treatment for individuals in asymptomatic stage of HIV infection or the pre-AIDS stage is very important. 


\section{Conflict of Interests}

The authors declare that there is no conflict of interests regarding the publication of this paper.

\section{Acknowledgments}

This work was partially supported by the NNSF of China (11461041), the NSF of Gansu Province of China (2013GS09485, 1107RJZA088), the NSF for Distinguished Young Scholars of Gansu Province of China (1111RJDA003), the Special Fund for the Basic Requirements in the Research of University of Gansu Province of China, and the Development Program for HongLiu Distinguished Young Scholars in Lanzhou University of Technology.

\section{References}

[1] World Health Organization, HIV/AIDS, http://www.who.int/ topics/hiv_aids/en/.

[2] N. Kaur, M. Ghosh, and S. S. Bhatia, "Modeling the spread of HIV in a stage structured population: effect of awareness," International Journal of Biomathematics, vol. 5, no. 5, Article ID 1250040, 2012.

[3] HIV and AIDS in South Africa, http://www.avert.org/aidssouthafrica.htm.

[4] H. F. Huo and L. X. Feng, "Global stability for an HIV/AIDS epidemic model with different latent stages and treatment," Applied Mathematical Modelling, vol. 37, no. 3, pp. 1480-1489, 2012.

[5] A. S. Perelson and P. W. Nelson, "Mathematical analysis of HIV1 dynamics in vivo," SIAM Review, vol. 41, no. 1, pp. 3-44, 1999.

[6] J. Lou, Y. Lou, and J. Wu, "Threshold virus dynamics with impulsive antiretroviral drug effects," Journal of Mathematical Biology, vol. 65, no. 4, pp. 623-652, 2012.

[7] R. P. Walensky, A. D. Paltiel, E. Losina et al., "The survival benefits of AIDS treatment in the United States," The Journal of Infectious Diseases, vol. 194, no. 1, pp. 11-19, 2006.

[8] L. Cai, X. Li, M. Ghosh, and B. Guo, "Stability analysis of an HIV/AIDS epidemic model with treatment," Journal of Computational and Applied Mathematics, vol. 229, no. 1, pp. 313-323, 2009.

[9] X. Lin, H. W. Hethcote, and P. van den Driessche, "An epidemiological model for HIV/AIDS with proportional recruitment," Mathematical Biosciences, vol. 118, no. 2, pp. 181-195, 1993.

[10] D. Li and W. Ma, "Asymptotic properties of a HIV-1 infection model with time delay," Journal of Mathematical Analysis and Applications, vol. 335, no. 1, pp. 683-691, 2007.

[11] X. Tian and R. Xu, "Global stability and Hopf bifurcation of an HIV-1 infection model with saturation incidence and delayed CTL immune response," Applied Mathematics and Computation, vol. 237, pp. 146-154, 2014.

[12] S. Bonhoeffer, J. M. Coffin, and M. A. Nowak, "Human immunodeficiency virus drug therapy and virus load," Journal of Virology, vol. 71, no. 4, pp. 3275-3278, 1997.

[13] Z. Yuan and X. Zou, "Global threshold dynamics in an hiv virus model with nonlinear infection rate and distributed invasion and production delays," Mathematical Biosciences and Engineering, vol. 10, no. 2, pp. 483-498, 2013.

[14] H. F. Huo and R. Chen, "Modelling the effect of treatment on an HIV/AIDS epidemic model," submitted.
[15] T. T. Yusuf and F. Benyah, "Optimal strategy for controlling the spread of HIV/AIDS disease: a case study of South Africa," Journal of Biological Dynamics, vol. 6, no. 2, pp. 475-494, 2012.

[16] H.-F. Huo, S.-J. Dang, and Y.-N. Li, "Stability of a two-strain tuberculosis model with general contact rate," Abstract and Applied Analysis, vol. 2010, Article ID 293747, 31 pages, 2010.

[17] P. van den Driessche and J. Watmough, "Reproduction numbers and sub-threshold endemic equilibria for compartmental models of disease transmission," Mathematical Biosciences, vol. 180, pp. 29-48, 2002.

[18] M. Bani-Yaghoub, R. Gautam, Z. Shuai, P. van den Driessche, and R. Ivanek, "Reproduction numbers for infections with free-living pathogens growing in the environment," Journal of Biological Dynamics, vol. 6, no. 2, pp. 923-940, 2012.

[19] G. H. Li and Z. Jin, "Global stability of a SEIR epidemic model with infectious force in latent, infected and immune period," Chaos, Solitons and Fractals, vol. 25, no. 5, pp. 1177-1184, 2005.

[20] Y. Cheng, J. Wang, and X. Yang, "On the global stability of a generalized cholera epidemiological model," Journal of Biological Dynamics, vol. 6, no. 2, pp. 1088-1104, 2012.

[21] J. P. LaSalle, The Stability of Dynamical Systems, vol. 25 of Regional Conference Series in Applied Mathmatics, SIAM, 1976.

[22] R. Xu, "Global stability of an HIV-1 infection model with saturation infection and intracellular delay," Journal of Mathematical Analysis and Applications, vol. 375, no. 1, pp. 75-81, 2011.

[23] J. Q. Li, Y. L. Yang, and Y. C. Zhou, "Global stability of an epidemic model with latent stage and vaccination," Nonlinear Analysis: Real World Applications, vol. 12, no. 4, pp. 2163-2173, 2011.

[24] J. Q. Li, Z. A. Ma, and F. Q. Zhang, "Stability analysis for an epidemic model with stage structure," Nonlinear Analysis: Real World Applications, vol. 9, no. 4, pp. 1672-1679, 2008. 


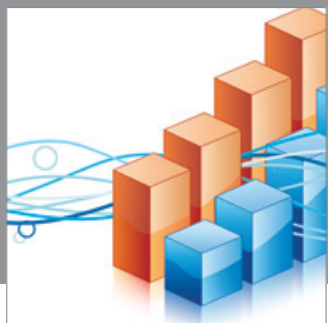

Advances in

Operations Research

mansans

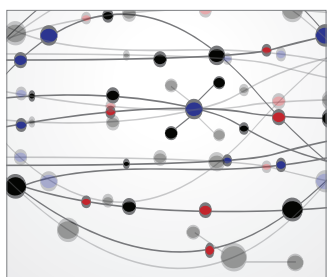

The Scientific World Journal
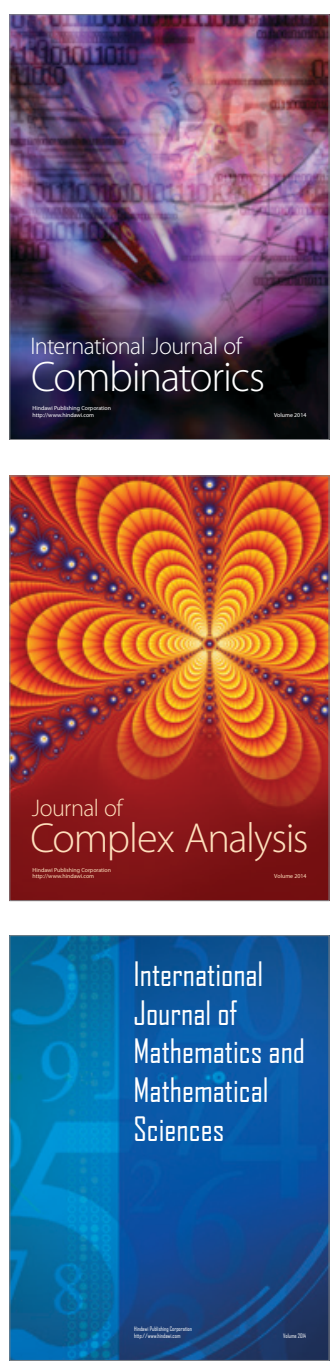
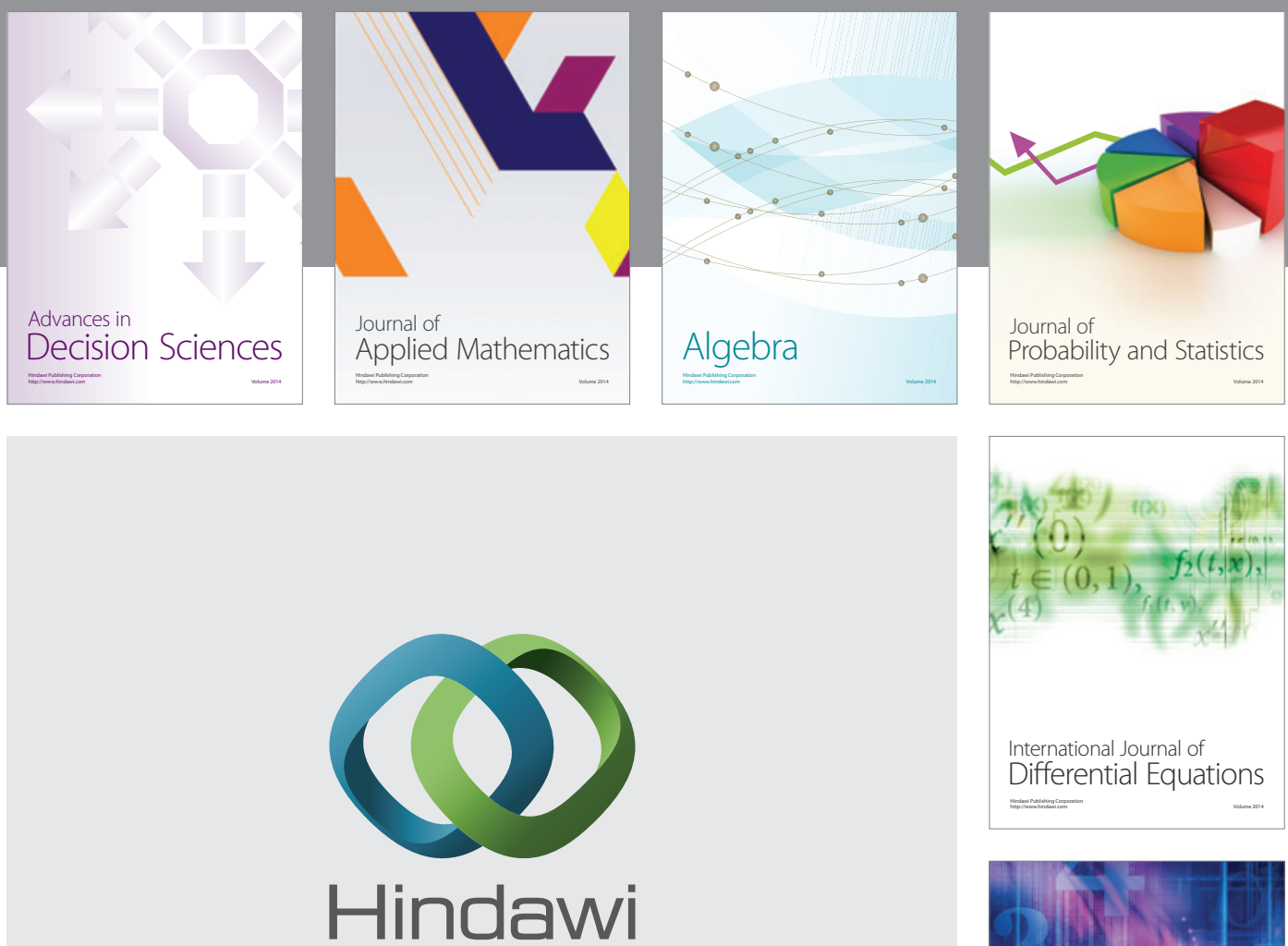

Submit your manuscripts at http://www.hindawi.com
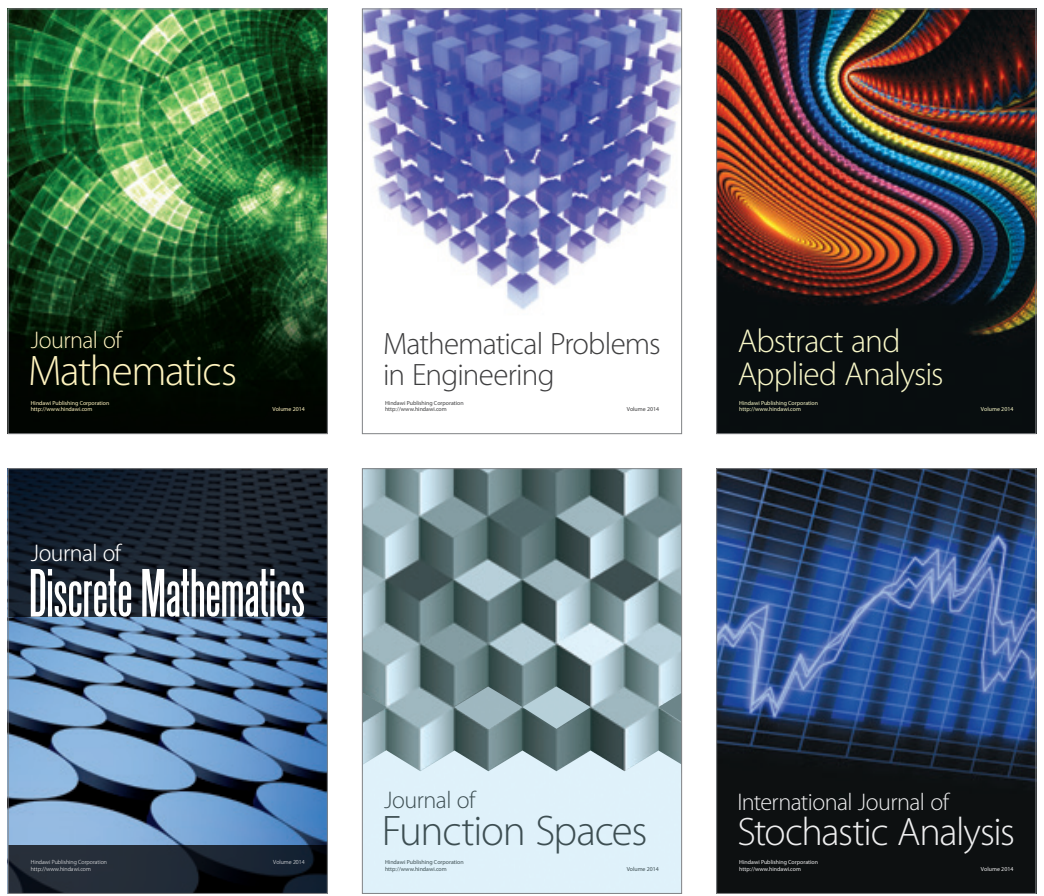

Journal of

Function Spaces

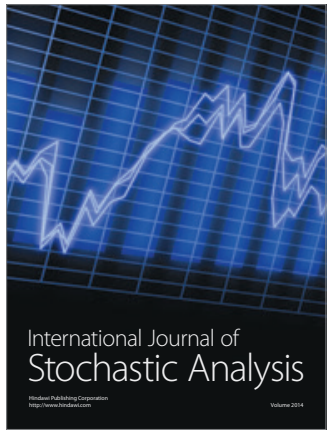

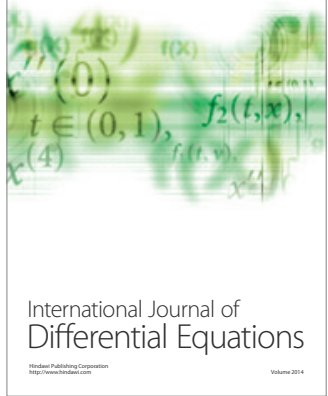
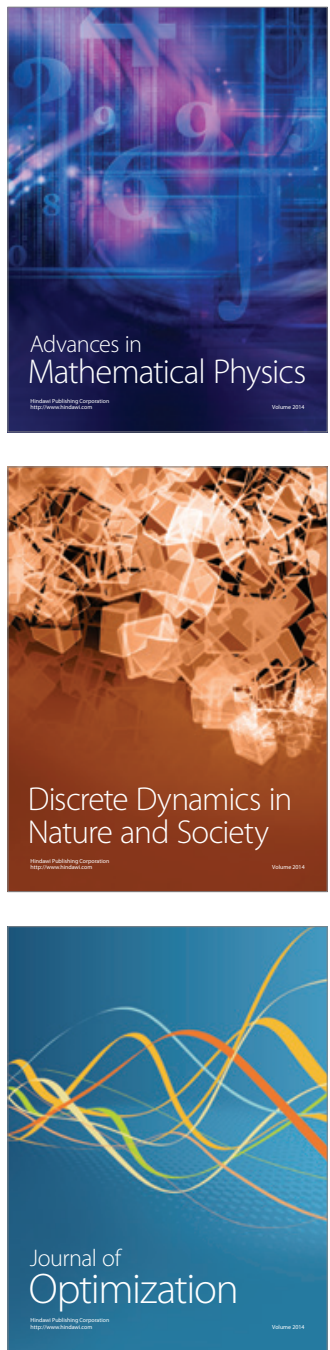